\title{
Modulated magnetization depth profile in dipolarly coupled magnetic multilayers
}

\author{
S. Bedanta, ${ }^{1}$ E. Kentzinger, ${ }^{2}$ O. Petracic, ${ }^{1, *}$ W. Kleemann,,${ }^{1, \dagger}$ U. Rücker, ${ }^{2}$ Th. Brückel, ${ }^{2}$ A. Paul, ${ }^{2}$ S. Cardoso, ${ }^{3}$ and \\ P. P. Freitas ${ }^{3}$ \\ ${ }^{1}$ Angewandte Physik, Universität Duisburg-Essen, 47048 Duisburg, Germany \\ ${ }^{2}$ Institut für Festkörperforschung, Forschungszentrum Jülich, 52425 Jülich, Germany \\ ${ }^{3}$ INESC, Rua Alves Redol 9-1, 1000 Lisbon, Portugal
}

(Received 10 July 2006; published 22 August 2006)

\begin{abstract}
Polarized neutron reflectivity (PNR) and magnetometry studies have been performed on the metal-insulator multilayer $\left[\mathrm{Co}_{80} \mathrm{Fe}_{20}(1.6 \mathrm{~nm}) / \mathrm{Al}_{2} \mathrm{O}_{3}(3 \mathrm{~nm})\right]_{9}$ which exhibits dominant dipolar coupling between the ferromagnetic layers. Our PNR measurements at the coercive field reveal a novel and unexpected magnetization state of the sample, exhibiting an oscillating magnetization depth profile from $\mathrm{CoFe}$ layer to $\mathrm{CoFe}$ layer with a period of five bilayers along the multilayer stack. With the help of micromagnetic simulations we demonstrate that competition between long- and short-ranged dipolar interactions apparently gives rise to this unprecedented phenomenon.
\end{abstract}

DOI: 10.1103/PhysRevB.74.054426

PACS number(s): 75.60.Jk, 61.12.Ha, 73.21.Ac, 78.20.Ls

Magnetic multilayers (MLs) consisting of ferromagnetic (FM) and nonmagnetic $(\mathrm{N})$ layers exhibit a large variety of physically interesting properties for both practical applications and fundamental research. In particular, magnetic multilayers comprising $3 d$ ferromagnetic layers interleaved with nonmagnetic spacers exhibit giant magnetoresistance for appropriate thicknesses of the spacer layers. ${ }^{1}$ Several types of interlayer coupling have been studied theoretically and experimentally (e.g., Ref. 2). For trilayers consisting of two magnetically saturated, pinhole-free metallic layers separated by a conductive spacer layer, the Ruderman-Kittel-KasuyaYosida (RKKY) oscillatory exchange provides a major coupling mechanism. In systems with insulating spacer layers and flat interfaces, dipolar interactions are dominant. It favors antiparallel orientation of the intraplanar magnetization between adjacent layers. For rough interfaces the so-called Néel- or orange peel interlayer coupling has to be considered. ${ }^{3,4}$ In 1962, Néel ${ }^{3}$ pointed out that there should be ferromagnetic coupling between adjacent films due to magnetic dipoles at the interface induced by a correlated morphological corrugation. Finally, if one considers the domain structure within each layer, a magnetostatic interaction could arise between the domain-wall stray fields in a $F M / N / F M$ structure, where $N$ can be a nonmagnetic metallic or insulating layer. Recently Lew et al..$^{5}$ have shown that interlayer domain-wall coupling can induce a mirror domain structure in a magnetic trilayer system which can affect the transport properties.

In this paper, we report on the observation of a novel magnetization state in a dipolar coupled magnetic metalinsulator multilayer exhibiting an oscillating magnetization depth profile from FM layer to FM layer. The paper is organized as follows: First we argue qualitatively how such unusual magnetization states can be induced by competing dipolar interactions. Then we present the experimental evidence from polarized neutron reflectometry. And, finally, a micromagnetic simulation is presented which underpins the argument that competing interactions can lead to the observed oscillating magnetization depth profile.

Dipolar interaction can lead to frustration effects in magnetic multilayers with in-plane magnetization direction. Gen- erally, the long-ranged dipolar fields from one layer to the nearest layer and to the next-nearest layer will favor antiparallel magnetization. Since the nearest-neighbor interaction usually prevails, an antiparallel alignment throughout the multilayer stack will be stabilized. However, for rough interfaces, the ferromagnetic Néel interlayer coupling ${ }^{3,4}$ must be introduced in addition. Since the next-nearest neighboring layers remain antiferromagnetically coupled, a competing interaction can be encountered, which might give rise to modulated incommensurate phases. ${ }^{6}$ This has been shown, e.g., for Ising models with competing nearest- and next-nearestneighbor interactions (axial next-nearest-neighbor Ising, or so-called ANNNI models). As will be shown below, our system behaves similarly when superimposing a homogeneous magnetic field, which provides matching conditions.

Metal-insulator multilayers of $\left[\mathrm{Co}_{80} \mathrm{Fe}_{20}\left(t_{\mathrm{n}}=1.6 \mathrm{~nm}\right) /\right.$ $\left.\mathrm{Al}_{2} \mathrm{O}_{3}(3 \mathrm{~nm})\right]_{9}$ were prepared by Xe-ion beam sputtering on glass substrates. ${ }^{7}$ The nominal $\mathrm{CoFe}$ layer thickness $t_{\mathrm{n}}=1.6 \mathrm{~nm}$ warrants intraplanar percolation, hence, exchange-dominated ferromagnetism in two dimensions, while discontinuous nanoparticular layers emerge at lower coverages due to the pronounced Volmer-Weber growth mode. ${ }^{8-10}$ It is known from related granular $\mathrm{Co} / \mathrm{Al}_{2} \mathrm{O}_{3}$ multilayers ${ }^{11}$ that the vertical arrangement of the clusters from plane to plane is not random but shows a growthinduced self-organization. Hence, correlated roughness of adjacent layers can be expected in our case-an essential prerequisite for ferromagnetic Néel coupling. ${ }^{3}$ Indeed, as shown previously for a nonpercolating $\mathrm{CoFe} / \mathrm{Al}_{2} \mathrm{O}_{3}$ multilayer, ${ }^{12}$ we find close coincidence of $\mathrm{x}$-ray specular small-angle reflectivity and longitudinal diffuse scattering [Fig. 1(a)], which clearly indicates strong vertical correlation in the multilayer stack. X-ray diffraction (XRD) scans under grazing incidence were taken to measure the specular and the diffuse $\mathrm{x}$-ray scattering using a Bruker-AXS D8 diffractometer with $\mathrm{Cu} \mathrm{K}_{\alpha}(\lambda=0.154 \mathrm{~nm})$ radiation. The scattered intensity is measured both in the specular $(\theta-2 \theta)$ and in the longitudinal-diffuse $\left(\theta-2 \theta+0.1^{\circ}\right)$ geometry, as shown in Fig. 1(a). Figure 1(b) shows the true-specular (=specular minus off-specular) reflectivity curve along with its fit. Simula- 


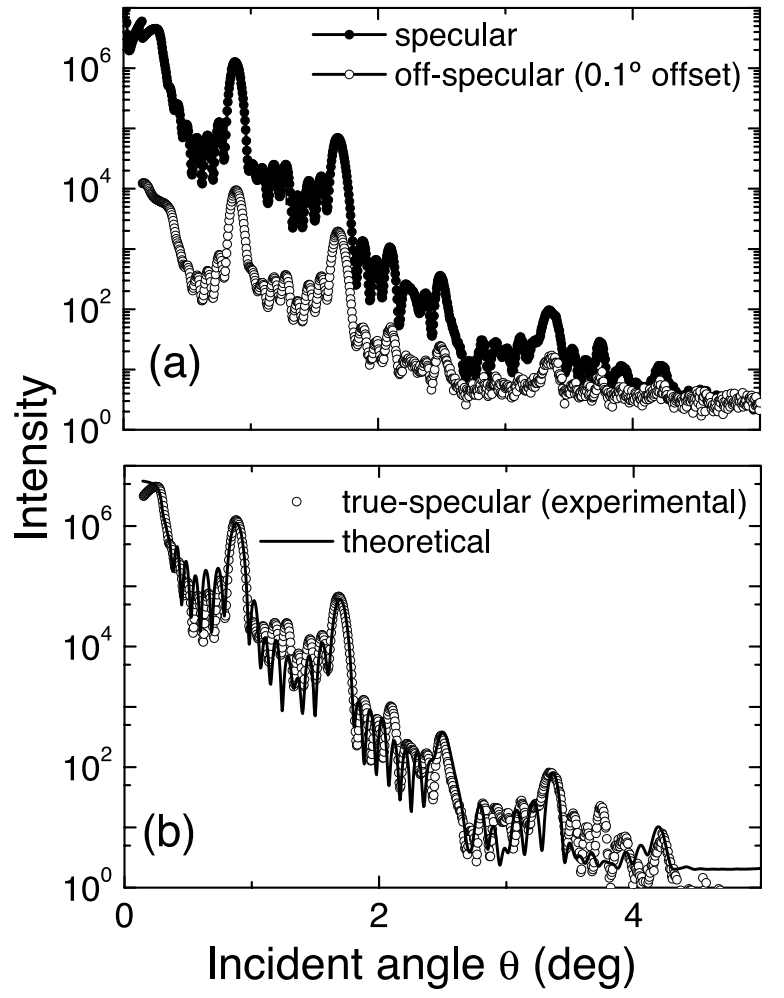

FIG. 1. (a) X-ray specular reflectivity and longitudinal diffuse scattering $\left(\theta-2 \theta+0.1^{\circ}\right)$ of a $\left[\mathrm{Co}_{80} \mathrm{Fe}_{20}(1.6 \mathrm{~nm}) / \mathrm{Al}_{2} \mathrm{O}_{3}(3 \mathrm{~nm})\right]_{9}$ multilayer. (b) The true-specular (see text) scan along with the best simulated curve for the multilayer is shown as a function of the angle of incidence.

tions of the true-specular curve (solid line) yield $1.36 \pm 0.02 \mathrm{~nm}$ and $3.67 \pm 0.02 \mathrm{~nm}$ for the thicknesses of the $\mathrm{CoFe}$ and $\mathrm{Al}_{2} \mathrm{O}_{3}$ layers, respectively, with a root-meansquare (rms) roughness of $0.42 \pm 0.02 \mathrm{~nm}$ for both $\mathrm{CoFe}$ and $\mathrm{Al}_{2} \mathrm{O}_{3}$.

During the growth of the sample, a magnetic field of $\mu_{0} H \approx 10 \mathrm{mT}$ was applied parallel to the sample plane, which leads to an in-plane easy axis in the sample. Magnetization hysteresis curves were measured by means of a superconducting quantum interference device (SQUID) magnetometer (MPMS-5S, Quantum Design). Figure 2 shows the dc moment $m$ vs the magnetic field $\mu_{0} H$ at $T=150 \mathrm{~K}$. The inset of Fig. 2 displays the magneto-optic longitudinal (open triangles) and polar (solid triangles) Kerr rotation (MOKE) at $T=300 \mathrm{~K}$. As can be seen, the polar component of magnetization in the sample is absent, i.e., the sample magnetization lies in the film plane.

Among the various experimental methods used to study the magnetic ordering in MLs, polarized neutron reflectometry (PNR) stands out by the ability to perform depthresolved, layer-by-layer magnetometry. Quantitative information on the laterally averaged layer magnetization as well as morphological parameters such as layer thicknesses, densities, or interface roughness can be obtained by fitting the specular reflectivity from the ML with a model. ${ }^{13}$ Offspecular or diffuse scattering gives additional information about lateral fluctuations such as the domain structure. Polarization analysis allows one to determine vector properties

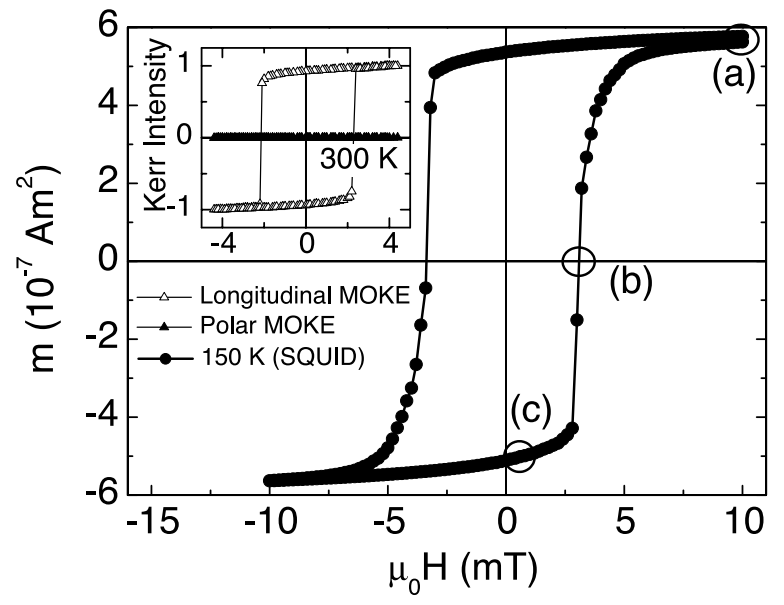

FIG. 2. Magnetic hysteresis of the ferromagnetically interacting nanoparticle system $\left[\mathrm{Co}_{80} \mathrm{Fe}_{20}(1.6 \mathrm{~nm}) / \mathrm{Al}_{2} \mathrm{O}_{3}(3 \mathrm{~nm})\right]_{9}$ at $T$ $=150 \mathrm{~K}$. The inset shows the normalized longitudinal (open triangles) and polar (solid triangles) MOKE intensity. The circles and labels (a), (b), and (c) mark the points on the hysteresis for which PNR measurements are shown in Fig. 3.

such as the angle of magnetization with respect to the neutron polarization or applied field direction.

We performed PNR measurements under small angles with the HADAS reflectometer at the Jülich research reactor FRJ-2 (DIDO). ${ }^{14}$ This instrument permits a simultaneous polarization analysis over a total range of scattering angles of $3^{\circ}$. We measured all four cross sections, $R^{++}, R^{--}, R^{+-}$, and $R^{-+}$for specular and off-specular (i.e., diffuse) conditions. The plus and minus signs indicate polarizations of the incident and scattered neutrons parallel or antiparallel to the applied external field, respectively. The non-spin-flip (NSF) data, $R^{++}$and $R^{--}$, depend on the chemical structure, as well as on the projection of the laterally averaged in-plane magnetization parallel to the neutron polarization. The spin-flip (SF) cross sections, $R^{+-}$and $R^{-+}$, arise solely from the projection of the in-plane magnetization perpendicular to the neutron polarization. We note that the efficiencies of the HADAS neutron polarizers were $>94 \%$ in external fields as small as $0.47 \mathrm{mT}$. Our PNR measurements were performed at $150 \mathrm{~K}$ with polarization analysis. At this temperature the coercive field is $\mu_{0} H \approx 3.1 \mathrm{mT}$, and the sample almost saturates at $12 \mathrm{mT}$ (Fig. 2). The wavelength of the neutron beam is $0.452 \mathrm{~nm}$, and the magnetic field is always applied parallel to the sample plane and to the easy axis.

Figure 3(a) shows PNR data taken at positive saturation $(12 \mathrm{mT})$. The FM state of the sample can be recognized by the splitting of the reflectivity edges where $R^{++}(\theta)$ is shifted to higher angles in comparison to $R^{--}(\theta) .^{12}$ The first-order structural superlattice Bragg peak at $\theta=2.45^{\circ}$ is dominant in the $R^{--}$channel. Its position corresponds to a bilayer thickness $d \approx 5.4 \mathrm{~nm}$, in agreement with the X-ray results (Fig. 1 ). The presence of a weak signal in the two SF channels $R^{+-}$ and $R^{-+}$is primarily due to the finite flipping ratio of 18 corresponding to the limited efficiency of $94 \%$ for the polarization analysis. The oscillatory behavior (Kiessig fringes) of $R^{++}$and $R^{--}$is due to the multilayer structure of the sample.

The PNR data shown in Fig. 3(c) were taken in the weak 

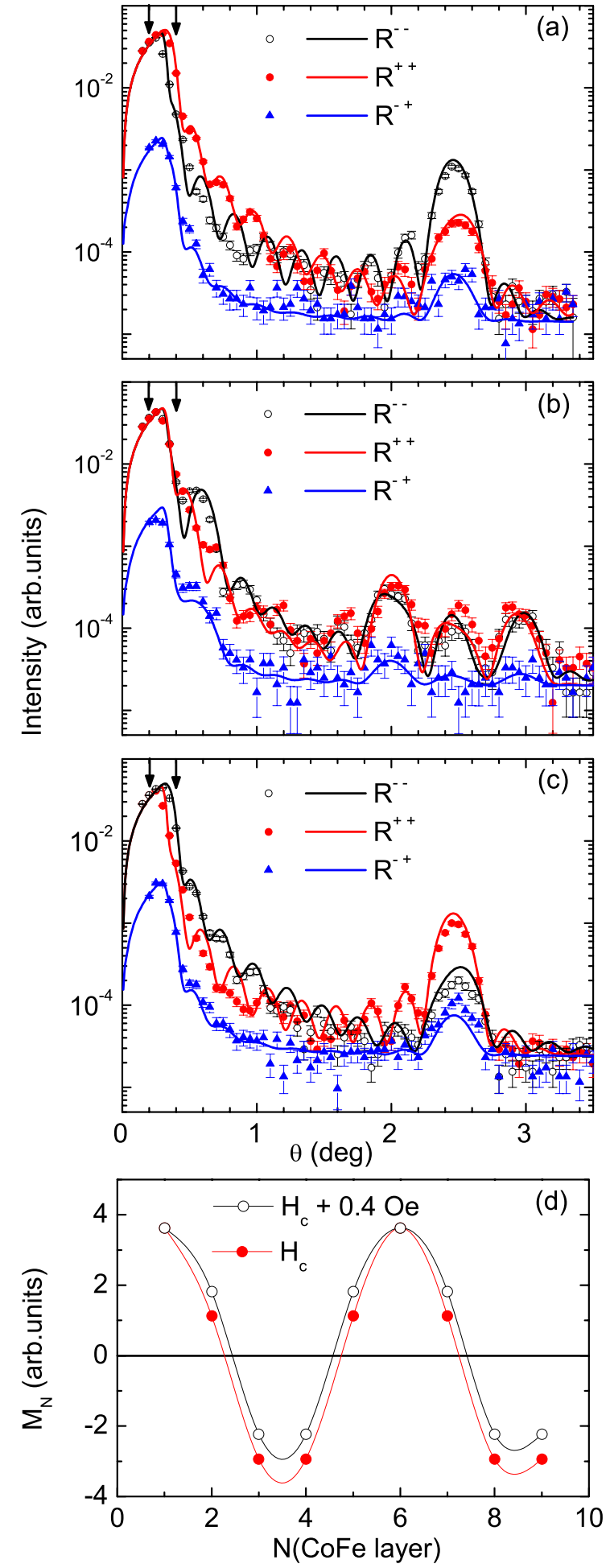

FIG. 3. (Color online) PNR reflectivities $R^{++}$in red (dark gray), $R^{--}$in black, and $R^{-+}$in blue (light gray) vs angle of incidence, $\theta$ measured at $T=150 \mathrm{~K}$ and $\mu_{0} H=12 \mathrm{mT}$ (saturation) (a), close to the coercive field $\mu_{0} H=3.8 \mathrm{mT}$ (b), and close to remanence in the guiding field of the neutrons, $\mu_{0} H=0.47 \mathrm{mT}$, after negative saturation (c) (see Fig. 2). The plateaus of total reflection, $Q_{\mathrm{c}}{ }^{++}$and $Q_{\mathrm{c}}{ }^{--}$, are designated by vertical arrows. (d) Magnetization of individual CoFe layers in the multilayer stack, numbered $N=1, \ldots, 9$ and extracted from the fitting to the PNR data at $H_{\mathrm{c}}$ (b, red), and to similar data for $\mu_{0} H_{\mathrm{c}}+0.04 \mathrm{mT}$ (black). guiding field $(0.47 \mathrm{mT})$ of the neutrons after negatively saturating the sample. They correspond to the negative remanence as confirmed by the small-angle data close to the plateau of total reflection, e.g., at $\theta=0.35^{\circ}$, where $R^{--}(\theta)>R^{++}(\theta)$. As there is no significant spin-flip scattering, there is definitely no magnetization component perpendicular to the applied field.

Figure 3(b) shows the PNR components measured at the positive coercive field after negatively saturating the sample. They correspond to the demagnetized state, as confirmed by the small-angle data close to the plateau of total reflection, where $R^{--}(\theta) \approx R^{++}(\theta)$. Interestingly two enhanced satellites evolve around the first structural superlattice Bragg peak. Here no significant spin flip scattering was observed, which proves the absence of any in-plane transverse magnetization component in the sample. The PNR measurements performed at $\mu_{0} H_{\text {appl }} \approx \mu_{0} H_{\mathrm{c}}+0.04 \mathrm{mT}$ also shows similar enhanced satellites around the first Bragg peak, except that the intensity of the $R^{++}$and $R^{--}$are slightly changed. The data are successfully fitted with a modulated magnetization depth profile from CoFe layer to layer with a periodicity of five layers [Fig. 3(d)]. This model is explained in terms of a multidomain state in each $\mathrm{CoFe}$ layer.

Unfortunately, we did not observe any off-specular scattering, from which one could estimate the average domain size in the sample. This might be due either to the limited scattering intensity or to the resolution limit of this PNR experiment, which cannot resolve domains being larger than $\xi \approx 30 \mu \mathrm{m} .{ }^{15}$ Further, it is worth mentioning that PNR measurements performed at various other points of the hysteresis loop never yield SF scattering, which hints at the absence of any in-plane transverse magnetization component in the sample. Finally, the occurrence of a modulated magnetization profile as evidenced by a splitting of the first structural superlattice Bragg peak has also been observed in related samples, e.g., at larger $\mathrm{CoFe}$ coverages in $\left[\mathrm{Co}_{80} \mathrm{Fe}_{20}(1.8 \mathrm{~nm}) / \mathrm{Al}_{2} \mathrm{O}_{3}(3 \mathrm{~nm})\right]_{9}$ (not shown). In this sample a period of approximately eight bilayers was found.

In order to understand the experimental observation of the magnetization reversal process in our multilayer system, micromagnetic simulations have been performed. We begin with the three-dimensional model within the object-oriented micromagnetic modeling framework (OOMMF) public micromagnetic code ${ }^{16}$ and extend the expressions of each of its energy terms to account for a variation in thickness from one cell to the next. ${ }^{17}$ The lateral sample size for the simulation is chosen to be $480 \mathrm{~nm} \times 480 \mathrm{~nm}$. We have taken nine magnetic and nine nonmagnetic layers in an alternative arrangement. The thickness is $3.2 \mathrm{~nm}$ for both magnetic and nonmagnetic layers. The cell size is $10 \times 10 \times 3.2 \mathrm{~nm}^{3}$. For the simulation, we assume a saturation magnetization of 1.44 MA/m, which corresponds to the value for a ferromagnetic Co layer. The roughness parameter being primarily due to the initial Volmer-Weber granular growth of the single layers $^{7-11}$ is mimicked by using a random modulation of the saturation magnetization, $M_{\mathrm{s}}($ cell $)=M_{\mathrm{s}}(\mathrm{Co})-\Delta M_{\mathrm{s}}$ $\times \operatorname{rand}(0,1)$, where $\operatorname{rand}(0,1)$ is a random number between 0 and 1 . In the present simulation we chose $\Delta M_{\mathrm{S}}=1.2 \mathrm{MA} / \mathrm{m}$. Two in-plane anisotropy terms are incorporated in the simu- 
(a)

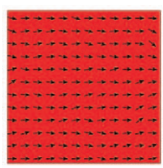

Layer 1

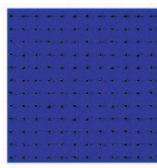

Layer 6

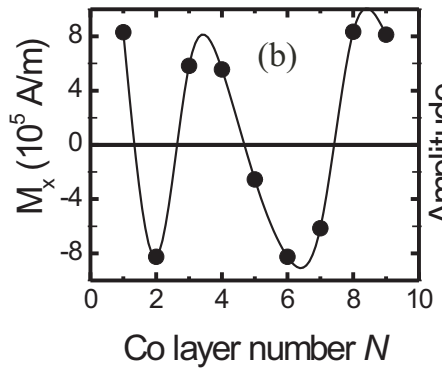

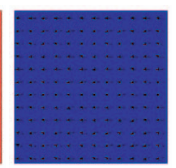

2

8

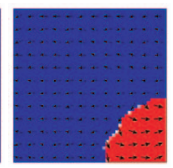

7

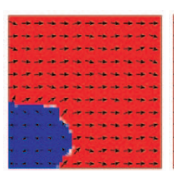

3

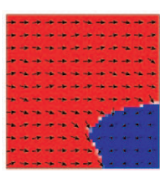

4
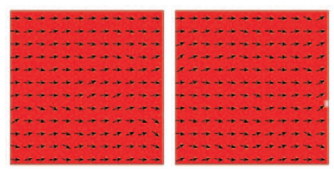

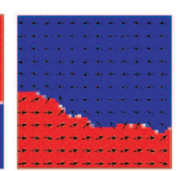

5

9

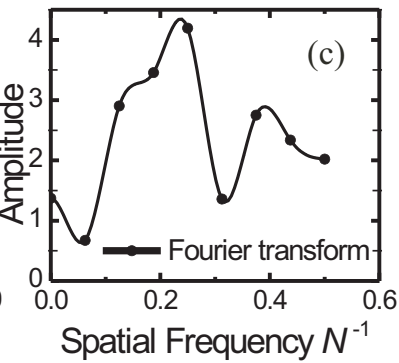

FIG. 4. (Color online) (a) Spin structure of the single layers coded $M_{\mathrm{x}}$ in red (light gray) and blue (dark gray) for $M_{\mathrm{x}}>0$ and $<0$, respectively, obtained from micromagnetic simulations (see text). The layer magnetization $M_{\mathrm{x}}$ vs distance in units of the bilayer thickness $d=6.4 \mathrm{~nm}$ and the corresponding Fourier transform in units $d^{-1}$ are shown in panels (b) and (c), respectively.

lation: (i) $K_{1}=0.05 \mathrm{MJ} / \mathrm{m}^{3}$, uniform uniaxial anisotropy expected for our experimental system, and (ii) $K_{\mathrm{r}}=0.1 \mathrm{MJ} / \mathrm{m}^{3}$, random uniaxial anisotropy in order to simulate the still present granularity of the film. The dominant interaction between the layers is dipolar interaction. Néel interlayer coupling is realized by the roughness in the magnetic layers. The equilibrium magnetization was found by solving the LandauLifshitz-Gilbert equation. ${ }^{18}$ Figure 4(a) shows the magnetization spin structure in each Co layer near the coercive field. In Fig. 4(b), the x component of magnetization for each Co layer is shown versus its number $N, 1 \leqslant N \leqslant 9$. It is seen that the Co layers are not just oppositely magnetized but display intermediate magnetized states. The simulation results are in agreement with the model adapted to the PNR data [Fig. 3(d)]. As mentioned earlier, there are mainly two kinds of interactions present in the simulation: the long-ranged dipolar interaction and the Néel coupling due to the roughness of the layers. In zero external magnetic field the latter interaction obviously dominates and stabilizes the global ferromagnetic state of the multilayer. However, when applying a reverse field the ferromagnetic interaction is weakened with respect to the dipolar next-nearest-neighbor antiferromagnetic interaction. That is why the multilayer experiences close to the coercive field a kind of ANNNI interaction scheme, where the incommensurate magnetization modulation is observed both in the experiment and in the numerical simulation. If the simulation results for the magnetization were described by a fundamental sinusoidal variation, we obtain a periodicity of $4.2 d$ ( $d=$ bilayer thickness), in rough agreement with the observed value of $\approx 5 d$. This is corroborated by a formal calculation of the Fourier transform of the magnetization in reciprocal space, which yields a peak at about $0.24 d^{-1}$ [Fig. 4(c)].

In summary, by polarized neutron reflectivity we have observed oscillating magnetization depth profiles in purely dipolarly coupled multilayers. In the demagnetized state periodicities of several bilayers (approximately five and eight, respectively) have been found in two different multilayer stacks. Similar results have emerged from micromagnetic simulations showing that the competition between dipolar and Néel coupling can indeed lead to stable domain states with oscillatory net magnetization from layer to layer. Future research should be aimed at exploring the essential ingredients which determine the incommensurate wave number.

Financial support by the Deutsche Forschungsgemeinschaft via Graduate School 277 "Structure and Dynamics of Heterogeneous Systems" and Grant KL306/38-2 is gratefully acknowledged.
*Present address: Department of Physics (EXSS), Imperial College London, London SW7 2AZ, United Kingdom.

Corresponding author. Email address: kleemann@uni-duisburg.de

${ }^{1}$ M. N. Baibich, J. M. Broto, A. Fert, F. Nguyen Van Dau, F. Petroff, P. Etienne, G. Creuzet, A. Friederich, and J. Chazelas, Phys. Rev. Lett. 61, 2472 (1988); G. Binasch, P. Grunberg, F. Saurenbach, and W. Zinn, Phys. Rev. B 39, R4828 (1989).

${ }^{2}$ P. Bruno, Phys. Rev. B 52, 411 (1995).

${ }^{3}$ L. Néel, Acad. Sci., Paris, C. R. 255, 1676 (1962).

${ }^{4}$ B. D. Schrag, A. Anguelouch, S. Ingvarsson, G. Xiao, Y. Lu, P. L. Trouilloud, A. Gupta, R. A. Wanner, W. J. Gallagher, P. M. Rice, and S. S. P. Parkin, Appl. Phys. Lett. 77, 2373 (2000); S. Tegen, I. Mönch, J. Schumann, H. Vinzelberg, and C. M. Schneider, J. Appl. Phys. 89, 8169 (2001).

${ }^{5}$ W. S. Lew, S. P. Li, L. Lopez-Diaz, D. C. Hatton, and J. A. C. Bland, Phys. Rev. Lett. 90, 217201 (2003).
${ }^{6}$ P. Bak, Rep. Prog. Phys. 45, 587 (1982); W. Selke, Phys. Rep. 170, 213 (1998).

${ }^{7}$ G. N. Kakazei, Yu. G. Pogorelov, A. M. L. Lopes, J. B. Sousa, S. Cardoso, P. P. Freitas, M. M. Pereira de Azevedo, and E. Snoeck, J. Appl. Phys. 90, 4044 (2001).

${ }^{8}$ W. Kleemann, O. Petracic, Ch. Binek, G. N. Kakazei, Yu. G. Pogorelov, J. B. Sousa, S. Cardoso, and P. P. Freitas, Phys. Rev. B 63, 134423 (2001).

${ }^{9}$ S. Sahoo, O. Petracic, W. Kleemann, S. Stappert, G. Dumpich, P. Nordblad, S. Cardoso, and P. P. Freitas, Appl. Phys. Lett. 82, 4116 (2003).

${ }^{10}$ X. Chen, S. Bedanta, O. Petracic, W. Kleemann, S. Sahoo, S. Cardoso, and P. P. Freitas, Phys. Rev. B 72, 214436 (2005).

${ }^{11}$ D. Babonneau, F. Petroff, J.-L. Maurice, F. Fettar, A. Vaurés, and A. Naudon, Appl. Phys. Lett. 76, 2892 (2000).

${ }^{12} \mathrm{~S}$. Bedanta, O. Petracic, E. Kentzinger, W. Kleemann, U. Rücker, 
A. Paul, Th. Brückel, S. Cardoso, and P. P. Freitas, Phys. Rev. B 72, 024419 (2005).

${ }^{13}$ J. F. Ankner and G. P. Felcher, J. Magn. Magn. Mater. 200, 741 (1999); C. F. Majkrzak, Physica B 221, 342 (1996); E. Kentzinger, U. Rücker, and B. P. Toperverg, Physica B 335, 82 (2003).

${ }^{14}$ U. Rücker, B. Alefeld, W. Bergs, E. Kentzinger, and Th. Brückel, Physica B 276-278, 95 (2000); U. Rücker, W. Bergs, B. Alefeld, E. Kentzinger, and Th. Brückel, Physica B 297, 140
(2001).

${ }^{15}$ A. Paul, E. Kentzinger, U. Rücker, D. E. Bürgler, and P. Grünberg, Eur. Phys. J. B 45, 249 (2005).

${ }^{16}$ M. J. Donahue and D. G. Porter, OOMMF User's Guide (National Institute of Standards and Technology, Gaithersburg, MD, 1999), Version 1.23.

${ }^{17}$ D. G. Porter and M. J. Donahue, J. Appl. Phys. 89, 7257 (2001).

${ }^{18}$ A. Aharoni, Introduction to the Theory of Ferromagnetism (Clarendon, Oxford, 1996). 